\title{
Design Construtal aplicado a um caminho condutivo em forma de seta inserido em um sólido com geração de calor
}

Constructal Design applied to an arrow-shaped conductive pathway intruded in a solid with heat generation

\author{
A. S. Barros ${ }^{1 *}$; E. A. Pinto Junior ${ }^{1}$; D. B. Santos ${ }^{1}$; F. K. Sell Junior ${ }^{1}$; R. G. \\ Hübner'; G. C. Rodrigues²; A. P. D. Aghenese ${ }^{2}$; C. M. B. Dutra' ;. A. \\ Teixeira $^{2}$; E. D. Santos ${ }^{1,2}$ \\ ${ }^{l}$ Programa de Pós-Graduação em Engenharia Oceânica - PPGEO, Universidade Federal do Rio Grande - FURG, \\ 96201-900, Rio Grande - RS, Brasil \\ ${ }^{2}$ Programa de Pós-Graduação em Modelagem Computacional - PPGMC, Universidade Federal do Rio Grande - \\ FURG, 96201-900, Rio Grande - RS, Brasil \\ *andreiabarros85@gmail.com
}

\begin{abstract}
Este trabalho busca avaliar geometricamente, através do Design Construtal, um caminho de alta condutividade a fim de melhor distribuir o campo de temperaturas diminuindo a maior temperatura observada em um domínio com geração de calor e de baixa condutividade. Para a análise numérica deste estudo, foi utilizado o software MATLAB junto com a ferramenta PDETOOL (Partial Differential Equation Toolbox). O caminho condutivo possui uma forma de seta, ou seja, composto por uma parte retangular com razão de medidas variáveis e uma parte triangular de área fixa. O problema passa a ter três restrições de área com seis variáveis, resultando em três graus de liberdade, $H / L, H_{0} / L_{0}$ e $H_{1} / L_{1}$, sendo que apenas $H_{0} / L_{0}$ varia. As simulações numéricas foram feitas para três diferentes condutividades, considerando quatro valores de fração de área preestabelecidos. Foi possível obter a geometria do caminho condutivo que melhor distribui o campo de temperaturas, resultando na minimização da temperatura máxima. Observouse nas três diferentes condutividades que, quanto maior a distribuição da área ocupada pelo material de alta condutividade em relação à área da placa, melhor distribuída será a temperatura, resultando na redução da temperatura máxima observada. Constatou-se também que a mais baixa condutividade térmica analisada, apresentou a menor temperatura máxima à medida que a razão do grau de liberdade foi aumentando. Já a maior condutividade térmica analisada apresentou menor temperatura máxima à medida que a razão do grau de liberdade foi diminuindo.

Palavras-chave: Design Construtal. Caminho condutivo. Otimização geométrica. Transferência de Calor.
\end{abstract}

This work seeks to evaluate geometrically, through the Constructal Design, a pathway of high thermal conductivity in order to better distribute the temperature field decreasing the highest temperatures observed in a domain with heat generation and low conductivity. For the numerical analysis of this study, MATLAB software was used and PDETOOL (Partial Differential Equation Toolbox). The conductive pathway has an arrow-shaped form, consisting of a rectangular part with ratio of variable measures and a triangular part with fixed area. The problem has been submitted to three constraint areas with six variables, resulting in three degrees of freedom $H / L, H_{0} / L_{0}$ and $H_{1} / L_{1}$, only $H_{0} / L_{0}$ varies. The numerical simulations were made for three different thermal conductivities, considering four values of pre-established fraction area. It was possible to obtain the geometry of the conductive pathway that best distributes the temperature field, resulting in the minimization of the maximum temperatures. It was observed for the three conductivities that as higher is the occupancy area of the high conductive material better is the temperature distribution along the domain, resulting in the reduction of the maximum observed temperature. It was also found that the lowest thermal conductivity analyzed presented the smallest maximum temperature as the ratio of the degree of freedom was increasing. The higher thermal conductivity analyzed presented lower maximum temperature as the ratio of the degree of freedom decreased.

Keywords: Constructal Design, Conductive pathway, Geometric optimization, Heat transfer

\section{INTRODUÇÃO}

Uma das grandes aplicações das tecnologias de refrigeração é a indústria de dispositivos eletrônicos, pois sem o devido resfriamento não há como manter circuitos e sistemas eletrônicos 
funcionando perfeitamente. Devido a essa tendência de miniaturização e aumento das capacidades e potências dos dispositivos mais modernos, a necessidade de ferramentas capazes de trocar calor eficientemente cresce proporcionalmente. Para o funcionamento adequado, um chip precisa ser mantido abaixo de $85^{\circ} \mathrm{C}$ ou controlado pela temperatura especificada pelo fabricante $[1,2]$.

A busca pela melhoria no desempenho de sistemas motiva a engenharia ao estudo de diferentes mecanismos de transferência de calor e a otimização da geometria destes, criando métodos para obtê-los. Um dos métodos utilizados é o resfriamento por convecção, porém sua aplicação em circuitos eletrônicos é inapropriada, pois para controlar a temperatura de um sistema, utiliza-se canais de transferência de calor que ocupam muito espaço. Por isso é necessário construir estruturas de condução de calor com materiais de elevada condutividade, assim o calor pode ser trocado com o meio de forma elevada e rápida $[3,4]$.

Uma forma de avaliar geometricamente dispositivos eletrônicos, com o intuito de minimizar a máxima temperatura gerada pelo mesmo, é através da simulação numérica empregando o Design Construtal [5, 6]. De acordo com Bejan e Zane (2013) [7], a lei Construtal foi apresentada pela primeira vez em 1995 e passou a ser aplicada em diversos problemas que envolvem fluxo, essa lei estabelece que para um sistema de fluxo de tamanho finito persistir no tempo, em outras palavras, para que ele sobreviva, sua configuração geométrica deve evoluir de tal maneira que facilite as correntes que fluem através dele. Assim, o Design Construtal é o método baseado na lei Construtal, utilizado para obter geometrias que facilitem o fluxo ou escoamento em um sistema. Esse método é fundamentado no princípio de objetivos e restrições, ou seja, estabelecido um objetivo para o problema, como minimizar a máxima temperatura ou maximizar a potência, são definidas as restrições, como área, temperatura, velocidade etc., e então são estabelecidos os graus de liberdade do problema, que irão variar alterando a geometria do sistema com o intuito de alcançar o objetivo preestabelecido.

O estudo desenvolvido por Hajmohammadi et al. (2013) [8] propôs um tipo simples de cavidade, que consiste em $\mathrm{N}$ ramos de cavidades de resfriamento inseridas em um corpo retangular com geração de calor. Realizou-se um processo de otimização detalhado que leva à minimização do pico de temperatura em um corpo gerador de calor, otimizando as cavidades de $\mathrm{N}$ ramos em relação às restrições de volume. Concluiu-se que aumentando o número de ramos, o pico de temperatura é minimizado pelas cavidades e ao comparar com cavidades do tipo complexo ( T, Y, TY ou H), encontradas na literatura, foram obtidos melhores resultados. Portanto, os autores indicam que as cavidades de $\mathrm{N}$ ramos devem ser priorizadas para fins de resfriamento prático.

No estudo realizado por Lorenzini et al. (2016) [5] foi aplicado Design Construtal sobre uma via de alta condutividade em forma de I que é inserida em um meio volumétrico com baixa condutividade térmica e geração de calor. Um terceiro material foi adicionado entre o meio condutor e o meio gerador de calor que atua como resistência ao contato térmico. Com o objetivo de encontrar geometrias que facilitem o fluxo de calor, diminuindo os pontos de maior temperatura do local onde está localizado. Os resultados obtidos indicam que a resistência de contato térmico é maior quando a condutividade térmica do material de alta condutividade e a condutividade térmica do material que representa a resistência de contato térmico são menores. Podendo aumentar a temperatura máxima e a proporção ideal em $56 \%$ e $17 \%$, respectivamente.

Posteriormente, Lorenzini et al. (2017) [6] aplicaram Design Construtal para avaliar numericamente a configuração de uma via em formato de $\mathrm{T}$ com alta condutividade inserida em um meio volumétrico gerador de calor com baixa condutividade térmica. Ainda é inserido outro material entre a via condutora e o meio gerador de calor que funciona como uma resistência de contato térmico. A finalidade é minimizar o excesso máximo de temperatura em todo o sistema, ou seja, reduzir os pontos quentes de um sistema com geração de calor uniforme. Os resultados indicam que para a geometria otimizada, a presença de resistência de contato térmico alterou as relações de comprimento e largura ideais em cerca de $16 \%$ e $12 \%$ em comparação com as relações obtidas com um perfeito contato térmico.

A presente pesquisa utiliza o Design Construtal para avaliar numericamente a geometria de um caminho bi-geométrico de alta condutividade térmica inserido em um volume de baixa condutividade térmica com geração de calor constante. A função do caminho altamente condutivo é resfriar o volume ao facilitar o fluxo de calor para fora do sistema. O objetivo da simulação é 
minimizar a máxima temperatura do sistema, mantendo o volume total do domínio e do caminho de alta condutividade constantes, variando, porém, a geometria do caminho condutivo. Como simplificação, o problema é avaliado bidimensionalmente e o caminho de alta condutividade térmica é dividido em duas áreas, uma com formato retangular e outra com formato triangular, onde apenas a primeira terá variação em seus parâmetros geométricos. Assim, foram determinadas três restrições de áreas e seis variáveis para o problema, resultando em três graus de liberdade, sendo dois constantes e um variável. O grau de liberdade variável é representado pela razão entre a altura e a base da área retangular que compõe o caminho condutivo.

Para as simulações numéricas foram estipuladas três condutividades, quatro valores para a razão entre a área do caminho de alta condutividade térmica e a área total do sistema. Para cada razão de área foi construído um intervalo de graus de liberdade a partir de um valor máximo e outro mínimo, aos quais o caminho condutivo está limitado por conta da geometria do sistema. São simulados todos os graus de liberdade pertencentes ao intervalo construído para cada razão de área e então obtidas as máximas temperaturas. Com isso foi possível avaliar a configuração geométrica que melhor minimiza a temperatura máxima do sistema.

\section{MODELAGEM MATEMÁTICA E NUMÉRICA}

O domínio bidimensional utilizado para a análise numérica do problema é representado na Figura 1. Trata-se de um problema de transferência de calor por condução em regime permanente em que um domínio de alta condutividade é inserido em um corpo gerador de calor. O corpo gerador de calor é uma placa bidimensional de área $A\left(\mathrm{~m}^{2}\right)$ que possui condutividade térmica constante $\mathrm{K}(\mathrm{W} / \mathrm{m} . \mathrm{K})$, enquanto que o elemento de alta condutividade é representado pela área $\left(A_{0}+A_{1}\right)\left(\mathrm{m}^{2}\right)$ que não possui geração de calor e tem condutividade térmica $k_{0}(\mathrm{~W} / \mathrm{m} . \mathrm{K})$ maior do que a da área $A$. As superfícies externas da placa com geração de calor são perfeitamente isoladas, com exceção da região inicial do caminho condutivo que possui temperatura prescrita inferior à temperatura da placa $T_{\text {mín. }}$

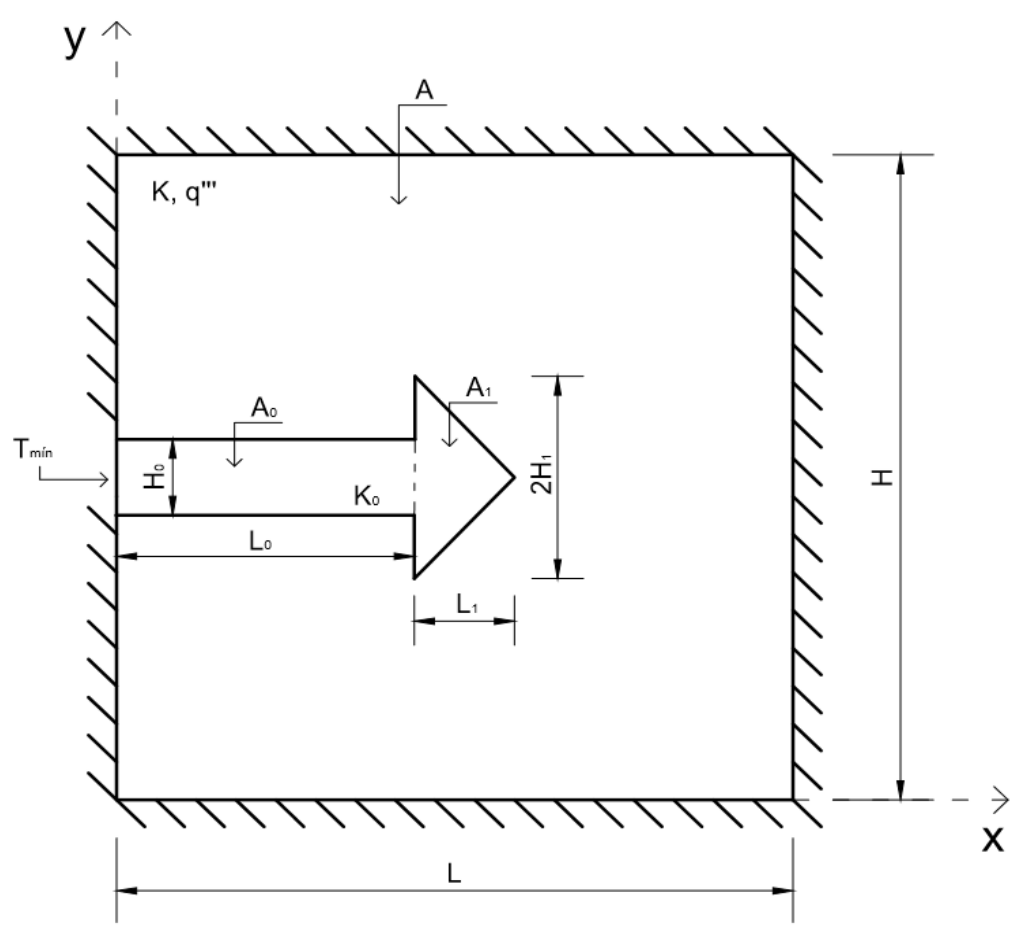

Figura 1: Domínio computacional do problema proposto.

De acordo com Incropera et al. (2008) [9], como há transferência de calor por condução em regime permanente com geração de calor uniforme e condutividade térmica constante na região da placa, a condução térmica, neste caso, pode ser representada pela Eq. (1). 
$\frac{\partial^{2} T}{\partial x^{2}}+\frac{\partial^{2} T}{\partial y^{2}}+\frac{q^{\prime \prime \prime}}{k_{p}}=0$

Segundo Lorenzini et al. (2016) [5], os seguintes grupos adimensionais podem ser empregados na solução desse problema:

$$
\begin{aligned}
& \tilde{T}=\frac{\left(T-T_{\min }\right)}{\left(q^{\prime \prime \prime} A / k_{p}\right)} \\
& K=\frac{k_{0}}{k_{p}}
\end{aligned}
$$

$\tilde{x}, \tilde{y}, \widetilde{H}, \widetilde{L}, \widetilde{H_{0}}, \widetilde{L_{0}}, \widetilde{H_{1}}, \widetilde{L_{1}}=\frac{x, y, H, L, H_{0}, L_{0}, H_{1}, L_{1}}{A^{1 / 2}}$

Assume-se que nenhum circuito (componente eletrônico) é disposto sobre o material de alta condutividade térmica, de modo que não há geração de calor na área ocupada por este material, sendo assim, a equação adimensional da transferência de calor é representada por:

$$
\frac{\partial^{2} \tilde{T}}{\partial \tilde{x}^{2}}+\frac{\partial^{2} \tilde{T}}{\partial \tilde{y}^{2}}=0
$$

A temperatura máxima adimensional é representada na Eq. (6) [5]:

$$
\tilde{T}_{\max }=\frac{\left(T_{\max }-T_{\min }\right)}{\left(q^{\prime \prime \prime} A / k_{p}\right)}
$$

Além disso, a Eq. (6) também é a resistência térmica entre a placa e o ponto onde ela está sendo resfriada.

O caminho de alta condutividade tem como objetivo minimizar a temperatura máxima adimensional do circuito, de modo que a configuração da geometria varie mantendo as três áreas constantes. Pode-se dizer que o material de alta condutividade inserido na placa possui duas áreas, uma delas tem formado retangular denotada por $\mathrm{A}_{0} \mathrm{e}$ a outra tem formato triangular e é denotada por $\mathrm{A}_{1}$. Dessa forma, existem três restrições de área neste problema que são representadas pelas seguintes equações:

$$
\begin{aligned}
& A=H \cdot L \\
& A_{0}=H_{0} \cdot L_{0} \\
& A_{1}=H_{1} \cdot L_{1}
\end{aligned}
$$

Em termos adimensionais, obtém-se:

$$
\frac{A}{A}=\frac{H \cdot L}{A}=\frac{H}{A^{1 / 2}} \cdot \frac{L}{A^{1 / 2}}=\widetilde{H} \cdot \breve{L}=1
$$




$$
\begin{aligned}
& \frac{A_{0}}{A}=\frac{H_{0} \cdot L_{0}}{A}=\frac{H_{0}}{A^{1 / 2}} \cdot \frac{L_{0}}{A^{1 / 2}}=\widetilde{H}_{0} \cdot \widetilde{L}_{0} \\
& \frac{A_{1}}{A}=\frac{H_{1} \cdot L_{1}}{A}=\frac{H_{1}}{A^{1 / 2}} \cdot \frac{L_{1}}{A^{1 / 2}}=\widetilde{H}_{1} \cdot \widetilde{L}_{1}
\end{aligned}
$$

Definindo a fração de área ocupada pelo material de alta condutividade:

$$
\begin{gathered}
\phi_{0}=\frac{A_{0}}{A} \\
\phi_{1}=\frac{A_{1}}{A}
\end{gathered}
$$

Dessa forma, considerando as Eqs. (11) e (12), as Eqs. (13) e (14) podem ser reescritas, respectivamente, como:

$$
\begin{aligned}
& \phi_{0}=\widetilde{H}_{0} \cdot \widetilde{L}_{0} \\
& \phi_{1}=\widetilde{H}_{1} \cdot \widetilde{L}_{1}
\end{aligned}
$$

Assim, fica evidente que o problema possui três restrições, que são representadas pelas Eqs. (10), (13) e (14), e seis variáveis, sendo elas $H, L, H_{0}, L_{0}, H_{1}$ e $L_{1}$. Isso resulta em três graus de liberdade $H / L, H_{0} / L_{0}$ e $H_{1} / L_{1}$, destes, porém, para o problema em questão, o único que possui variação é $H_{0} / L_{0}$, já que os demais graus de liberdade possuem valores constantes.

Para a avaliação geométrica, são empregadas as seguintes equações:

$$
\begin{aligned}
& \tilde{L}_{0}=\left[\frac{\phi_{0}}{\left(H_{0} / L_{0}\right)}\right]^{\frac{1}{2}} \\
& \widetilde{H}_{0}=\left(\frac{\widetilde{H}_{0}}{\widetilde{L}_{0}}\right) \tilde{L}_{0}
\end{aligned}
$$

A modelagem numérica, utilizada para avaliar a geometria do material de alta condutividade, consiste na solução de um algoritmo implementado no software MATLAB R2012b com o auxílio da ferramenta PDE tool (Partial Differential Equation Toolbox) que foi empregada na geração da geometria e malha do domínio apresentado. A escolha da malha foi baseada no teste de independência de malha feito por Lorenzini et al. (2014) [10], que obteve o tamanho através de sucessivos refinamentos, utilizando elementos triangulares irregulares.

Foram feitas simulações numéricas para três diferentes condutividades, 5, 30 e 500 (W/m.K) e atribuiu-se valores constantes para a fração de área ocupada pelo material de alta condutividade na região onde se deseja fazer o estudo geométrico. Esses valores foram: 0,005, 0,010, 0,050 e 0,100 . As demais constantes admitidas no problema foram as seguintes: $H / L=1, H_{1} / L_{1}=4$ e $k_{0} / k_{\mathrm{p}}$ $=100$.

O algoritmo utilizado varia a geometria do caminho condutivo bi-geométrico conforme o valor atribuído à razão $H_{0} / L_{0}$, e retorna o valor da máxima temperatura adimensional observada no domínio. Durante as simulações para cada condutividade, para cada valor de $\phi_{0}$ preestabelecido foram atribuídos valores de $H_{0} / L_{0}$ a fim de determinar os limites máximos e mínimos aos quais o caminho condutivo estaria sujeito dentro do domínio. Com estes limites determinados, foi criado um intervalo de valores de $H_{0} / L_{0}$ igualmente espaçados, tendo como extremos os valores máximos 
e mínimos encontrados. Estes valores, inseridos no algoritmo, retornaram as respectivas temperaturas máximas resultantes de cada arranjo geométrico.

\section{RESULTADOS E DISCUSSÃO}

Com os parâmetros determinados e a variação do grau de liberdade, pode-se avaliar a geometria do caminho de alta condutividade presente no domínio com base na máxima temperatura gerada no mesmo.

Nas Figuras 2a e 2b é possível observar a malha gerada, bem como a geometria do caminho condutivo no limite mínimo e máximo, respectivamente, utilizando um $\phi_{0}$ de $0,005, H_{0} / L_{0}$ mínimo de 0,0055 e um $H_{0} / L_{0}$ máximo de 32 . O comportamento da malha foi igual para as três diferentes condutividades.

(a)

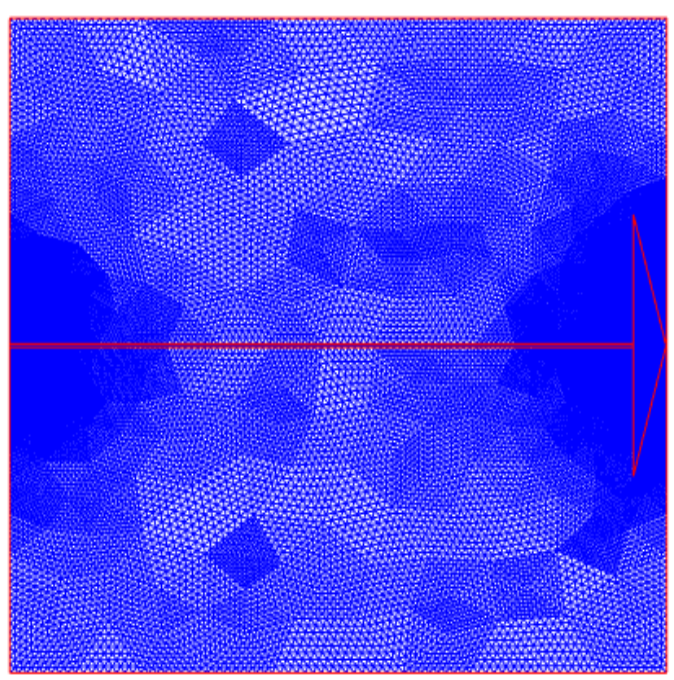

(b)

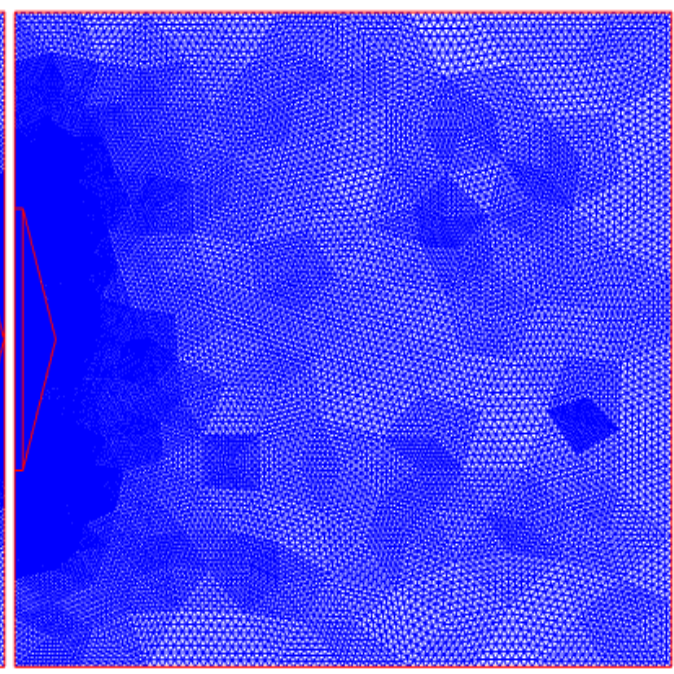

Figura 2: Geometria e malha gerada. (a) Para um $H_{0} / L_{0}=0,0055$. (b) Para um $H_{0} / L_{0}=32$.

Entre as três condutividades analisadas, o campo de temperaturas que tem a melhor distribuição foi na condutividade de 500 W/m.K, conforme apresentado nas Figuras 3a e 3b.

(a)

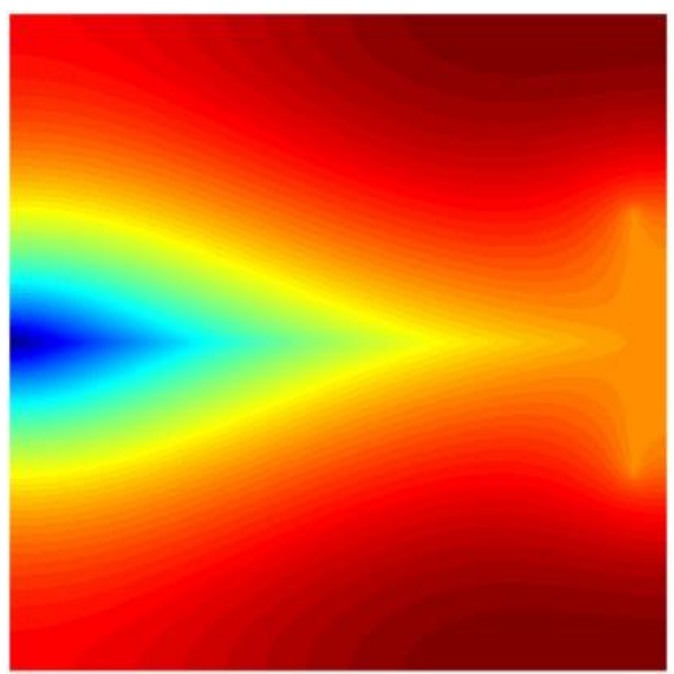

Figura 3: Campo de temperaturas. (a) Para um $H_{0} / L_{0}=0,0055$. (b) Para um $H_{0} / L_{0}=32$.

\section{(b)}

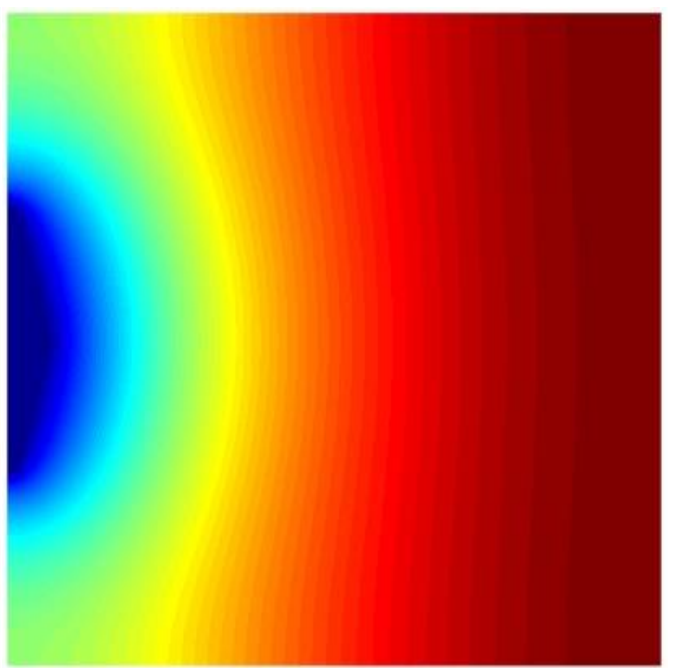

Pode-se observar na Figura 3b, que ao concentrar o material altamente condutivo à esquerda do domínio, a temperatura nessa região fica visivelmente mais baixa, o que é indicado pela cor 
azul, porém, como consequência, a temperatura máxima no lado oposto do domínio, aumenta consideravelmente.

Quando o caminho condutivo atravessa o domínio alcançando o limite máximo do lado direito, conforme observado na Figura 3a, permite uma melhor distribuição do campo de temperaturas. Essa configuração acarreta numa redução da maior temperatura máxima observada no domínio.

Com os resultados obtidos nas simulações foi possível construir o gráfico apresentado nas Figuras 4,5 e 6 , onde são demonstrados o efeito de $H_{0} / L_{0}$ sobre $T_{\text {máx }}$ para diferentes $\phi_{0}$. É possível observar nessas figuras que para as três condutividades analisadas, quanto maior a fração de área $\phi_{0}$, menor é a variação do grau de liberdade $H_{0} / L_{0}$ e maior a variação da temperatura máxima do domínio.

Na Figura 7 apresenta-se um gráfico onde é possível observar o efeito da fração de área $\phi_{0}$ sobre a temperatura máxima minimizada no domínio para as diferentes condutividades simuladas.

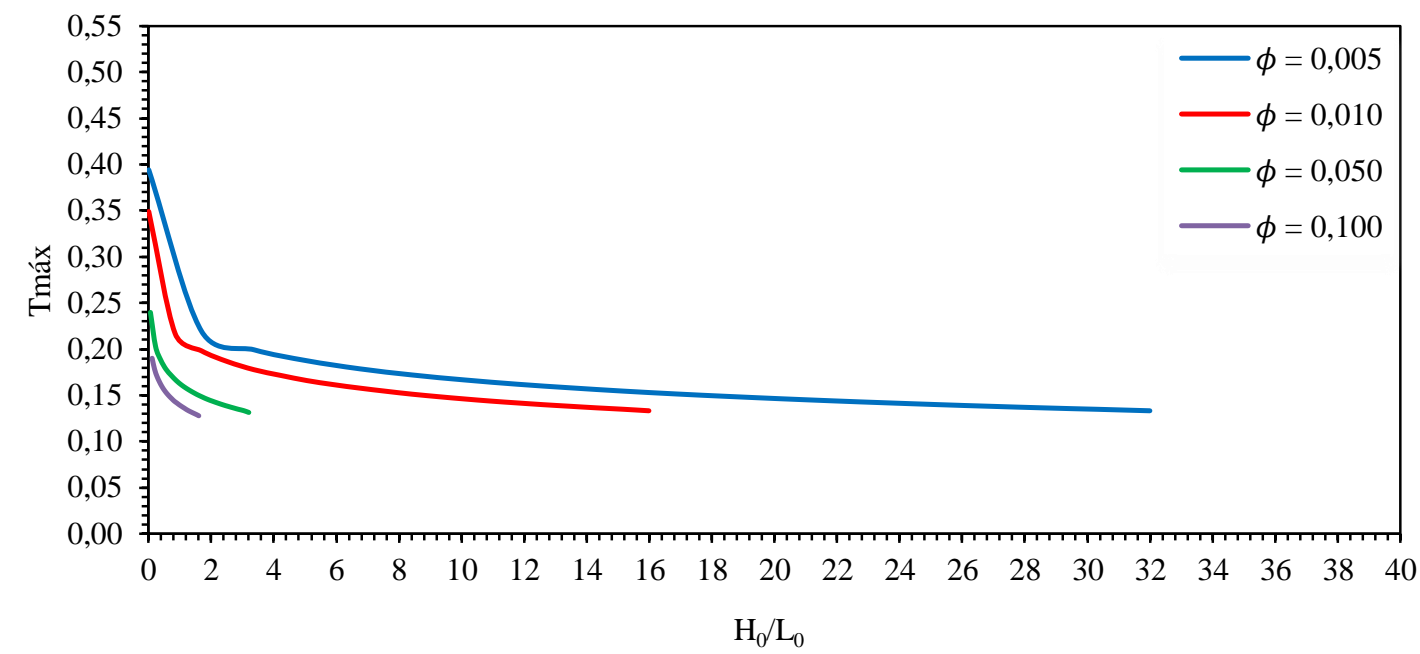

Figura 4: Efeito de $H_{0} / L_{0}$ sobre a temperatura máxima para diferentes $\phi_{0}$ e $K=5 \mathrm{~W} / \mathrm{m} . K$.

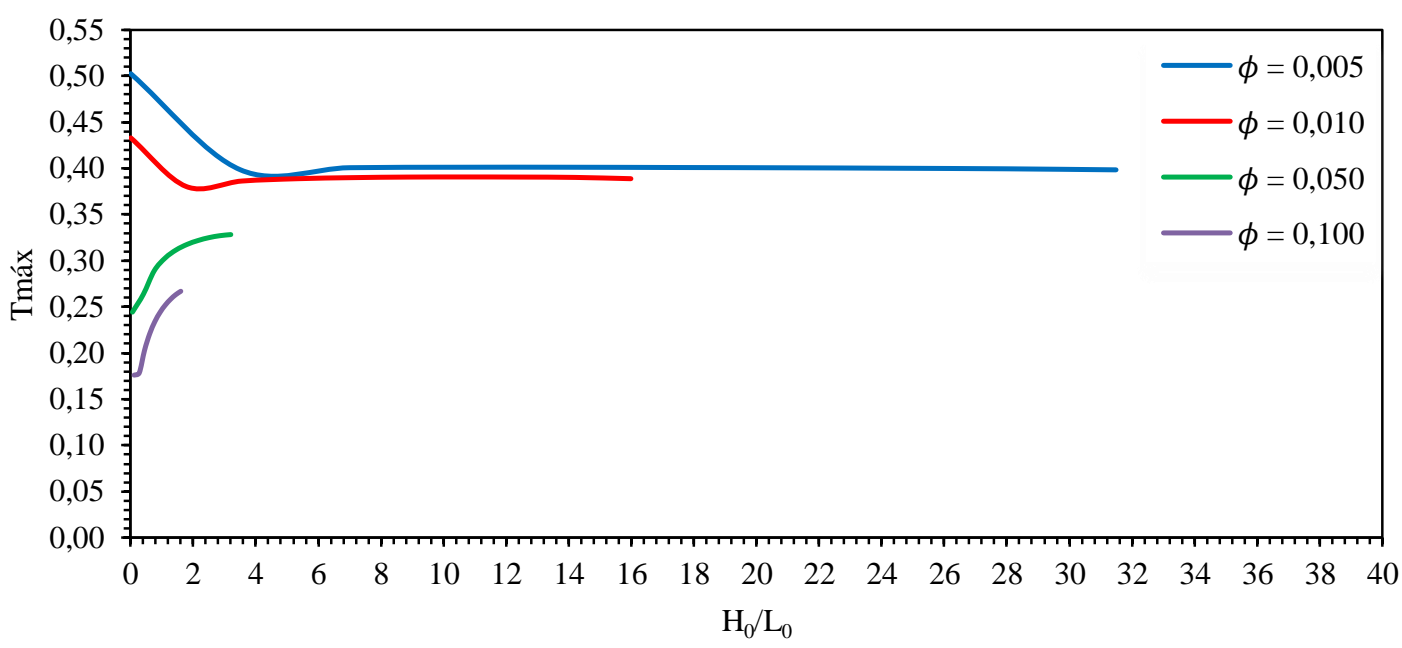

Figura 5: Efeito de $H_{0} / L_{0}$ sobre a temperatura máxima para diferentes $\phi_{0}$ e $K=30 \mathrm{~W} / \mathrm{m} . K$. 


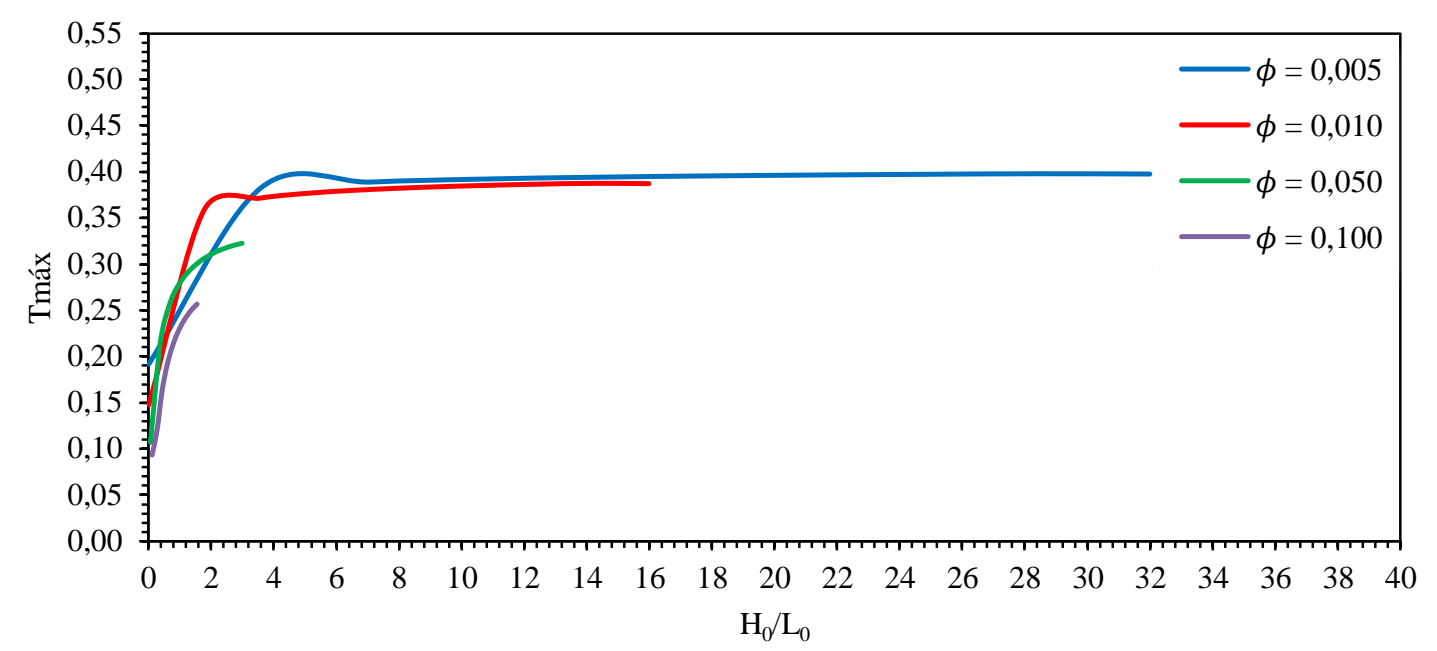

Figura 6: Efeito de $H_{0} / L_{0}$ sobre a temperatura máxima para diferentes $\phi_{0}$ e $k=500 \mathrm{~W} / \mathrm{m} . \mathrm{K}$.

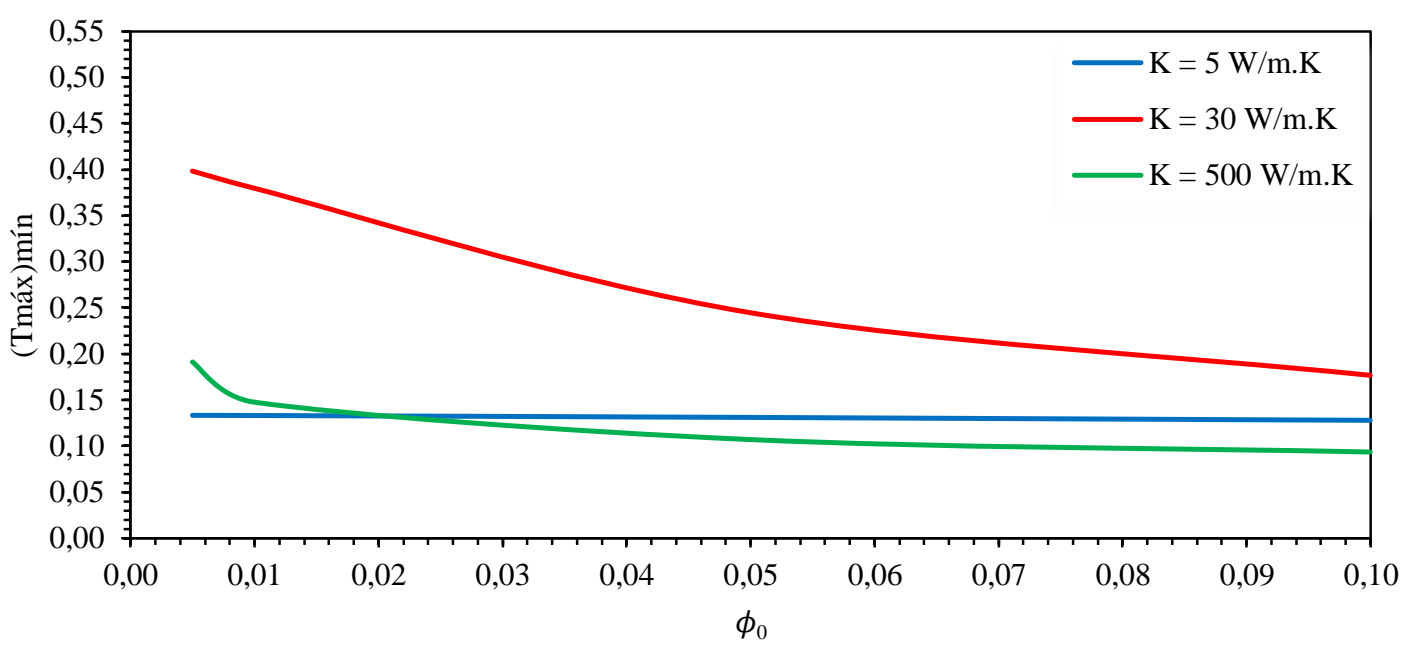

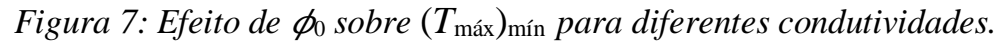

Observa-se na Figura 7 que, para as três condutividades, quanto maior a fração de área $\phi_{0}$, menor é $\left(T_{\text {máx }}\right)_{\text {mín }}$ no domínio. É possível notar que $\left(T_{\text {máx }}\right)_{\text {mín }}$ para uma condutividade de $5 \mathrm{~W} / \mathrm{m}$.K sofre uma variação muito pequena ao longo do aumento de $\phi_{0}$. Para a condutividade de $30 \mathrm{~W} / \mathrm{m}$.K existem variações maiores, maiores variações ocorrem, sendo as mesmas suavizadas após uma fração de área de 0,05. Para uma condutividade de $500 \mathrm{~W} / \mathrm{m} . \mathrm{K}\left(\mathrm{T}_{\text {máx }}\right)_{\text {mín }}$ tende a se estabilizar a partir de uma fração de área de 0,05 .

É possível perceber, através da Figura 8, o efeito da fração de área $\phi$, sobre o grau de liberdade ótimo $\left(H_{0} / L_{0}\right)_{0}$ para as diferentes condutividades. Observa-se que para a condutividade de 5 $\mathrm{W} / \mathrm{m} . \mathrm{K}$, o grau de liberdade $\left(H_{0} / L_{0}\right)_{\circ}$ diminui à medida que a fração de área $\phi_{0}$ aumenta. Esta diminuição tende a estabilizar a partir de um $\phi_{0}=0,05$. Já o grau de liberdade variável na condutividade de $30 \mathrm{~W} / \mathrm{m} . \mathrm{K}$, após sofrer uma diminuição considerável até um valor de $\phi_{0}$ aproximadamente igual a 0,025, aumenta levemente e tende a estabilizar a partir de um $\phi_{0}=0,04$. Por fim, para uma condutividade de $500 \mathrm{~W} / \mathrm{m}$.K, o grau de liberdade aumenta com uma variação muito baixa, à medida que a fração de área também aumenta.

É apresentado na Figura 9 o efeito de diferentes condutividades térmicas $K$ sobre $\left(T_{\text {máx }}\right)_{\text {mín }}$ o grau de liberdade ótimo $\left(H_{0} / L_{0}\right)_{\mathrm{o}}$ e a fração de área ótima $\phi_{0}$. É possível notar nessa figura que para todas as condutividades térmicas simuladas, é a maior fração de área que minimiza a temperatura máxima do sistema. Observa-se também que tanto a temperatura máxima ótima 
quanto a variação do grau de liberdade ótimo sofrem uma estabilização a partir da condutividade térmica de $60 \mathrm{~W} / \mathrm{m} . \mathrm{K}$.

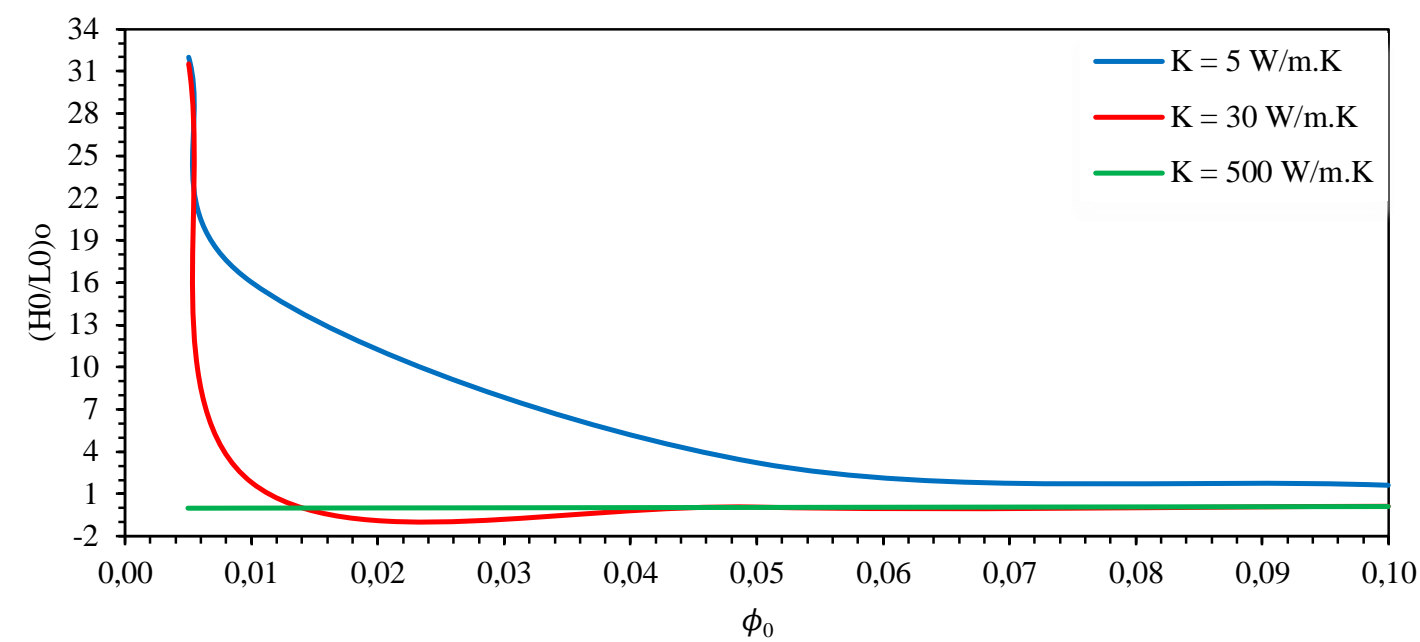

Figura 8: Efeito de $\phi_{0}$ sobre $\left(H_{0} / L_{0}\right)_{0}$ para diferentes condutividades.

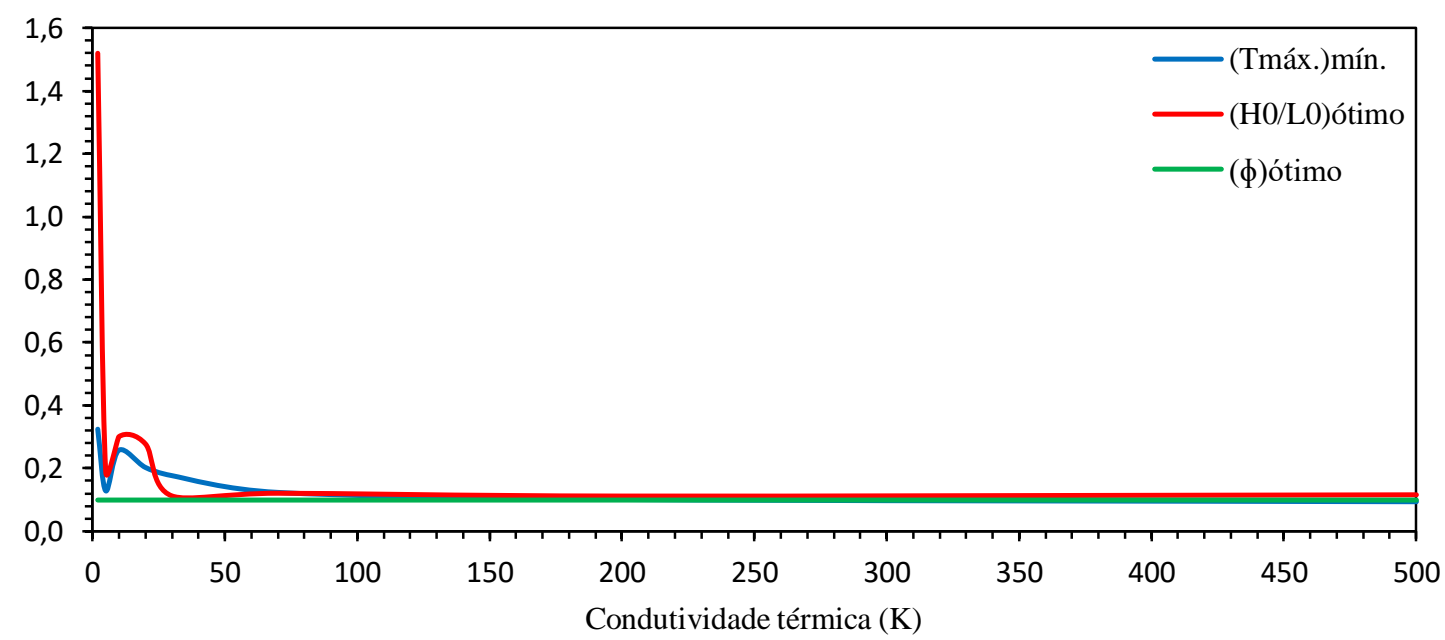

Figura 9: Efeito da condutividade térmica sobre $\left(T_{\text {máx }}\right)_{\text {mín, }}\left(H_{0} / L_{0}\right)_{0}$ e $\phi_{0}$.

\section{CONCLUSÃO}

Este trabalho utilizou o método do Design Construtal para analisar geometricamente a distribuição do campo de temperaturas em um sistema com geração de calor, contendo um caminho bi-geométrico de alta condutividade sem geração de calor. $\mathrm{O}$ estudo buscou atingir a menor temperatura máxima do domínio. Utilizou-se o software MATLAB e a ferramenta PDE tool (Partial Differential Equation Toolbox) para a realização das simulações numéricas e solução do problema. Os graus de liberdade e geometrias foram mantidos fixos, exceto a relação $\mathrm{H}_{0} / \mathrm{L}_{0}$ que variou de acordo com os valores de $\phi_{0}$ previamente estabelecidos. As simulações foram feitas para diferentes condutividades térmicas, através das quais foi possível constatar que, quanto maior o $\phi_{0}$, menor a variação da temperatura e menor é a máxima temperatura minimizada do sistema, o que se pode ser observado facilmente na Figura 7.

Os resultados possibilitaram também, a análise do efeito da fração de área ocupada sobre a razão ótima para os três casos de condutividades térmicas, sendo constatado, através da Figura 8, 
que para estes três casos, $\left(H_{0} / L_{0}\right)_{0}$ são simultaneamente estáveis a partir de um $\phi_{0}$ equivalente à 0,05 .

Por fim, a Figura 9 mostrou o efeito de $\left(\mathrm{T}_{\text {máx }}\right)_{\min },\left(H_{0} / L_{0}\right)_{\circ}$ e $\phi_{0}$ para simulações de oito diferentes condutividades térmicas. Com esta análise, foi possível concluir que é a maior fração de área que minimiza a temperatura máxima do sistema. Foi possível observar também que tanto a temperatura máxima ótima quanto a variação do grau de liberdade ótimo, sofrem uma estabilização a partir da condutividade térmica de $60 \mathrm{~W} / \mathrm{m} . \mathrm{K}$. É interessante salientar que nem sempre obter a menor temperatura local em uma determinada região de um domínio é a melhor solução, pois a homogeneidade da dissipação térmica também é de extrema importância. Uma conclusão deve ser apresentada com as principais contribuições do estudo.

\section{AGRADECIMENTOS}

Os autores agradecem à Capes e ao $\mathrm{CNPq}$ pelo suporte financeiro que permitiu a realização desta pesquisa.

\section{REFERÊNCIAS BIBLIOGRÁFICAS}

1. Trisch M. Resfriamento de componentes eletrônicos por jatos sintéticos tangenciais. [Dissertação]. São Leopoldo (RS): Universidade do Vale do Rio do Sinos; 2015. 104 p.

2. Mathews RN, Balaji C. Numerical simulation of conjugate, turbulent mixed convection heat transfer in a vertical channel with discrete heat sources. Int Comm Heat Mass Transf. 2006 Aug;33(7):908-916, doi: 10.1016/j.icheatmasstransfer.2006.02.013.

3. Bejan A. Constructal-theory network of conducting paths for cooling a heat generating volume. Int $\mathbf{J}$ Heat Mass Transf. 1997 Mar;40(4):799-816, doi: 10.1016/0017-9310(96)00175-5.

4. Bejan A. Shape and Structure, from Engineering to Nature. Cambridge: Cambridge University Press; 2000. $362 \mathrm{p}$.

5. Lorenzini G, Barreto EX, Beckel CC, Schneider PS, Isoldi LA, Dos Santos ED, Rocha LAO. Constructal Design of I-shaped high conductive pathway for cooling a heat-generating medium considering the thermal contact resistance. Int J Heat Mass Transf. 2016 Feb;93(1):770-777, doi: 10.1016/j.ijheatmasstransfer.2015.10.015.

6. Lorenzini G, Barreto EX, Beckel CC, Schmeider PS, Isoldi LA, Dos Santos ED, Rocha LAO. Geometrical evaluation of T-shaped high conductive pathway with thermal contact resistance for cooling of heat-generating medium. Int J Heat Mass Transf. 2017 May;108(B):1884-1893, doi: 10.1016/j.ijheatmasstransfer.2017.01.008.

7. Bejan A, Zane JP. Design in nature: how the constructal law governs evolution in biology, physics, technology, and social organization. New York: Random House; 2013. 304 p.

8. Hajmohammadi MR, Poozesh S, Campo A, Nourazar SS. Valuable reconsideration in the constructal design of cavities. Energy Convers Manag. 2013 Feb;66(1):33-40, doi: 10.1016/j.enconman.2012.09.031.

9. Incropera FP, DeWitt DP, Bergman TL, Lavine AS. Fundamentos de Transferência de Calor e de Massa. Rio de Janeiro: Livros Técnicos e Científicos - LTC; 2008. 664 p.

10. Lorenzini G, Biserni C, Estrada ED, Isoldi LA, dos Santos ED, Rocha LAO. Constructal Design of Convective Y-Shaped Cavities by Means of Genetic Algorithm. J Heat Transf. 2014 Apr;136(7): 071702, doi: 10.1115/1.4027195. 